\title{
GENERALIZED SPHERICAL HARMONICS FOR CUBIC-TRICLINIC SYMMETRY
}

\author{
PETER R. MORRIS \\ 1276 Oakmont Drive, Hamilton, Ohio, OH 45013, USA
}

(Received 30 March 1995)

\begin{abstract}
An explicit representation is suggested for orthogonal generalized spherical harmonics with cubic-crystal and triclinic-sample symmetries. The representation employs sums and differences of orthogonal generalized spherical harmonics with cubic-crystal symmetry, previously described by Bunge for orthorhombic (or higher) sample symmetry, and is illustrated, for $\dot{\mathrm{T}}_{2}^{\mu \nu}, \imath=4,9, \mu=1, \nu=1$ to 5 . This representation facilitates crystallite orientation distribution (COD) analysis (aka ODF analysis) for these symmetries, using the Bunge formalism.
\end{abstract}

Keywords: Generalized spherical harmonics; Cubic-crystal symmetry; Triclinic-sample symmetry; COD; ODF

\section{INTRODUCTION}

Derivation of series representations of the crystallite orientation distribution from pole-figure measurements was suggested by Bunge (1965) and Roe (1965). The influence of cubic-crystal symmetry was considered by Bunge and Ehlert (1966), Roe (1966), and Esling et al. (1981). Summaries of the relevant mathematical treatments in German and in English are given in monographs by Bunge $(1962,1982)$. In his treatment, the superscript $\nu$ is used to index functions with appropriate sample symmetry, and he states, "The index $\nu$ will thereby enumerate in some sequence the linearly independent orthogonal solutions of this system of equations." He further assumes orthorhombic (or higher) sample symmetry.

Computer programs for cubic-crystal and triclinic-sample symmetries, using Roe's formalism, have been in use at Armco Research and 
Technology since 1976 (see Hook, 1993). In the following, an explicit representation is suggested for generalized spherical harmonics with cubic-crystal and triclinic-sample symmetries.

\section{SYMMETRY CONSIDERATIONS}

Bunge (1969), Eqs. (11.138)-(11.140), (1982), Eqs. (14.122)-(14.124), has indicated how cubic-crystal symmetry may be satisfied by linear combinations of the generalized spherical harmonics. The projectionoperator method for determination of the coefficients, $\dot{\mathrm{A}}_{\imath}^{\mathrm{m} \mu}$, of these combinations has been described by Esling et al. (1981). Bunge (1982) gives

$$
\dot{\mathrm{T}}_{\imath}^{\mu \nu}(\mathrm{g})=\sum_{\mathrm{n}=-\imath}^{+\imath} \stackrel{\bullet}{\mathrm{A}}_{\imath} \dot{\mathrm{T}}_{\imath}^{\mu n}(\mathrm{~g})
$$

The following expressions employing sums and differences of the $\dot{\mathrm{T}}_{2}^{\mu n}$ illustrate an explicit representation for generalized spherical harmonics with cubic-crystal and triclinic-sample symmetries. The $P_{\imath}^{m n}(\Phi)$ and real functions defined by Bunge (1982) Eqs. (14.85), (14.86) and Table 15.1.2., which can readily be calculated (Pospiech and Jura, 1975), providing sign is properly considered.

$$
\begin{aligned}
\dot{\mathrm{T}}_{4}^{11}= & \dot{\mathrm{T}}_{4}^{10}=\dot{\mathrm{A}}_{4}^{01} \mathrm{P}_{4}^{00}(\Phi)+2 \dot{\mathrm{A}}_{4}^{41} \mathrm{P}_{4}^{40}(\Phi) \cos 4 \phi_{2} \\
\dot{\mathrm{T}}_{4}^{12}= & \left(2^{-1 / 2}\right)\left(\dot{\mathrm{T}}_{4}^{1 \overline{1}}+\dot{\mathrm{T}}_{4}^{11}\right) \\
= & \mathrm{i} 2^{1 / 2}\left\{\dot{\mathrm{A}}_{4}^{01} \mathrm{P}_{4}^{01}(\Phi) \cos \phi_{1}\right. \\
& +\dot{\mathrm{A}}_{4}^{41}<\left[\mathrm{P}_{4}^{41}(\Phi)-\mathrm{P}_{4}^{41}(\pi-\Phi)\right] \cos 4 \phi_{2} \cos \phi_{1} \\
& \left.-\left[\dot{\mathrm{P}}_{4}^{41}(\Phi)+\mathrm{P}_{4}^{41}(\pi-\Phi)\right] \sin 4 \phi_{2} \sin \phi_{1}>\right\} \\
\dot{\mathrm{T}}_{4}^{13}= & \left(2^{-1 / 2}\right)\left(\dot{\mathrm{T}}_{4}^{1 \overline{1}}+\dot{\mathrm{T}}_{4}^{11}\right) \\
= & 2^{1 / 2}\left\{\dot{\mathrm{A}}_{4}^{01} \mathrm{P}_{4}^{01}(\Phi) \sin \phi_{1}\right.
\end{aligned}
$$


$+\dot{\mathrm{A}}_{4}^{41}<\left[\mathrm{P}_{4}^{41}(\Phi)-\mathrm{P}_{4}^{41}(\pi-\Phi)\right] \cos 4 \phi_{2} \sin \phi_{1}$

$\left.+\left[\mathbf{P}_{4}^{41}(\Phi)+\mathbf{P}_{4}^{41}(\pi-\Phi)\right] \sin 4 \phi_{2} \cos \phi_{1}>\right\}$

$$
\begin{aligned}
\dot{\mathrm{T}}_{4}^{14}= & \left(2^{-1 / 2}\right)\left(\dot{\mathrm{T}}_{4}^{1 \overline{2}}+\dot{\mathrm{T}}_{4}^{12}\right) \\
= & 2^{1 / 2}\left\{\dot{\mathrm{A}}_{4}^{01} \mathrm{P}_{4}^{02}(\Phi) \cos 2 \phi_{1}\right. \\
& +\dot{\mathrm{A}}_{4}^{41}<\left[\mathrm{P}_{4}^{42}(\Phi)+\mathrm{P}_{4}^{42}(\pi-\Phi)\right] \cos 4 \phi_{2} \cos 2 \phi_{1} \\
& \left.-\left[\mathrm{P}_{4}^{42}(\Phi)-\mathrm{P}_{4}^{42}(\pi-\Phi)\right] \sin 4 \phi_{2} \sin 2 \phi_{1}>\right\}
\end{aligned}
$$

$$
\begin{aligned}
\dot{\mathrm{T}}_{4}^{15} & =\left(2^{-1 / 2}\right)\left(\dot{\mathrm{T}}_{4}^{12}+\dot{\mathrm{T}}_{4}^{1 \overline{2}}\right) \\
& =\mathrm{i} 2^{1 / 2}\left\{\dot{\mathrm{A}}_{4}^{01} \mathrm{P}_{4}^{02}(\Phi) \sin 2 \phi_{1}\right. \\
& +\dot{\mathrm{A}}_{4}^{41}<\left[\mathrm{P}_{4}^{42}(\Phi)+\mathrm{P}_{4}^{42}(\pi-\Phi)\right] \cos 4 \phi_{2} \sin 2 \phi_{1} \\
& \left.+\left[\mathrm{P}_{4}^{42}(\Phi)-\mathrm{P}_{4}^{42}(\pi-\Phi)\right] \sin 4 \phi_{2} \cos 2 \phi_{1}>\right\}
\end{aligned}
$$

$$
\begin{aligned}
\ddot{\mathrm{T}}_{9}^{11}= & \dot{\mathrm{T}}_{9}^{10} \\
= & \mathrm{i} 2\left[\dot{\mathrm{A}}_{9}^{41} \mathrm{P}_{9}^{40}(\Phi) \sin 4 \phi_{2}+\dot{\mathrm{A}}_{9}^{81} \mathrm{P}_{9}^{80}(\Phi) \sin 8 \phi_{2}\right] \\
\ddot{\mathrm{T}}_{9}^{12}= & \left(2^{-1 / 2}\right)\left(\dot{\mathrm{T}}_{9}^{11}+\dot{\mathrm{T}}_{9}^{1 \overline{1}}\right) \\
= & -2^{1 / 2}\left\{\dot{\mathrm{A}}_{9}^{41}<\left[\mathrm{P}_{9}^{41}(\Phi)-\mathrm{P}_{9}^{41}(\pi-\Phi)\right] \cos 4 \phi_{2} \sin \phi_{1}\right. \\
& +\left[\mathrm{P}_{9}^{41}(\Phi)+\mathrm{P}_{9}^{41}(\pi-\Phi)\right] \sin 4 \phi_{2} \cos \phi_{1}> \\
& +\dot{\mathrm{A}}_{9}^{81}<\left[\mathrm{P}_{9}^{81}(\Phi)-\mathrm{P}_{9}^{81}(\pi-\Phi)\right] \cos 8 \phi_{2} \sin \phi_{1} \\
& \left.+\left[\mathrm{P}_{9}^{81}(\Phi)+\mathrm{P}_{9}^{81}(\pi-\Phi)\right] \sin 8 \phi_{2} \cos \phi_{1}>\right\}
\end{aligned}
$$

$$
\begin{aligned}
\dot{\mathrm{T}}_{9}^{13}= & \left(2^{-1 / 2}\right)\left(\dot{\mathrm{T}}_{9}^{11}-\dot{\mathrm{T}}_{9}^{1 \overline{1}}\right) \\
= & \mathrm{i} 2^{1 / 2}\left\{\dot{\mathrm{A}}_{9}^{41}<\left[\mathrm{P}_{9}^{41}(\Phi)-\mathrm{P}_{9}^{41}(\pi-\Phi)\right] \cos 4 \phi_{2} \cos \phi_{1}\right. \\
& -\left[\mathrm{P}_{9}^{41}(\Phi)+\mathrm{P}_{9}^{41}(\pi-\Phi)\right] \sin 4 \phi_{2} \sin \phi_{1}> \\
& +\dot{\mathrm{A}}_{9}^{81}<\left[\mathrm{P}_{9}^{81}(\Phi)-\mathrm{P}_{9}^{81}(\pi-\Phi)\right] \cos 8 \phi_{2} \cos \phi_{1} \\
& \left.-\left[\mathrm{P}_{9}^{81}(\Phi)+\mathbf{P}_{9}^{81}(\pi-\Phi)\right] \sin 8 \phi_{2} \sin \phi_{1}>\right\}
\end{aligned}
$$




$$
\begin{aligned}
\dot{\mathrm{T}}_{9}^{14}= & \left(2^{-1 / 2}\right)\left(\dot{\mathrm{T}}_{9}^{12}+\dot{\mathrm{T}}_{9}^{1 \overline{2}}\right) \\
= & \mathrm{i} 2^{1 / 2}\left\{\dot{\mathrm{A}}_{9}^{41}<\left[\mathrm{P}_{9}^{42}(\Phi)+\mathrm{P}_{9}^{42}(\pi-\Phi)\right] \cos 4 \phi_{2} \sin 2 \phi_{1}\right. \\
& +\left[\mathrm{P}_{4}^{42}(\Phi)-\mathrm{P}_{9}^{42}(\pi-\Phi)\right] \sin 4 \phi_{2} \cos 2 \phi_{1}> \\
& +\dot{\mathrm{A}}_{9}^{81}<\left[\mathrm{P}_{9}^{82}(\Phi)+\mathrm{P}_{9}^{82}(\pi-\Phi)\right] \cos 8 \phi_{2} \sin 2 \phi_{1} \\
& \left.+\left[\mathrm{P}_{9}^{82}(\Phi)-\mathrm{P}_{9}^{82}(\pi-\Phi)\right] \sin 8 \phi_{2} \cos 2 \phi_{1}>\right\} \\
\dot{\mathrm{T}}_{9}^{15}= & \left(2^{-1 / 2}\right)\left(\dot{\mathrm{T}}_{9}^{12}-\dot{\mathrm{T}}_{9}^{1 \overline{2}}\right) \\
= & 2^{1 / 2}\left\{\dot{\mathrm{A}}_{9}^{41}<\left[\mathrm{P}_{9}^{42}(\Phi)+\mathrm{P}_{9}^{42}(\pi-\Phi)\right] \cos 4 \phi_{2} \cos 2 \phi_{1}\right. \\
& -\left[\mathrm{P}_{9}^{42}(\Phi)-\mathrm{P}_{9}^{42}(\pi-\Phi)\right] \sin 4 \phi_{2} \sin 2 \phi_{1}> \\
& +\dot{\dot{\mathrm{A}}}_{9}^{81}<\left[\mathrm{P}_{9}^{82}(\Phi)+\mathrm{P}_{9}^{82}(\pi-\Phi)\right] \cos 8 \phi_{2} \cos 2 \phi_{1} \\
& \left.-\left[\mathrm{P}_{9}^{82}(\Phi)-\mathrm{P}_{9}^{82}(\pi-\Phi)\right] \sin 8 \phi_{2} \sin 2 \phi_{1}>\right\}
\end{aligned}
$$

\section{CONCLUSIONS}

An explicit representation for generalized spherical harmonics with cubic-crystal and triclinic-sample symmetries has been illustrated for $\imath=4,9, \mu=1, \nu=1,5$. Representations for other $\imath, \mu, \nu$ can be written from the illustrated examples.

\section{Acknowledgements}

I express my profound debt to the prior works of, and discourses over many years with, H. J. Bunge, C. Esling, J. Pospiech, and R. J. Roe.

\section{References}

Bunge, H. J. (1965), Z. Metalkde, 56, 872-4.

Bunge, H. J. (1969), Mathematische Methoden der Texturanalyse, Academie-Verlag, Berlin.

Bunge, H. J. (1982), Texture Analysis in Materials Science, Butterworths, London.

Bunge, H. J. and Ehlert, J. (1966), Mber. Dtsch. Akad. Wiss., 8, 241-59. 
Esling, C., Bechler-Ferry, E. and Bunge, H.J. (1981), Proc. 6th Int. Conf. on Textures of Materials, Tokyo, Sept 28-Oct. 3, 2, Iron and Steel Inst. of Japan, 1373-89.

Hook, R. E. (1993), Met. Trans. A, 24, 2009-19.

Pospiech, J. and Jura, J. (1975), Kristall und Technik, 10, 783-87.

Roe, R. J. (1965), J. Appl. Phys. 36, 2024-31.

Roe, R. J. (1966), J. Appl. Phys. 37, 2069-72. 